\title{
The specification of personalised insoles using additive manufacturing
}

\author{
André S. Salles ${ }^{* a}$ and Diane E. Gyi ${ }^{\mathrm{a}}$ \\ ${ }^{a}$ Loughborough Design School, Loughborough University, Loughborough, LE11 3TU, United Kingdom
}

\begin{abstract}
Research has been conducted to explore a process that delivers insoles for personalised footwear for the high street using additive manufacturing (AM) and to evaluate the use of such insoles in terms of discomfort. Therefore, the footwear personalisation process was first identified: (1) foot capture; (2) anthropometric measurements; (3) insole design; and (4) additive manufacturing. In order to explore and evaluate this process, recreational runners were recruited. They had both feet scanned and 15 anthropometric measurements taken. Personalised insoles were designed from the scans and manufactured using AM. Participants were fitted with footwear under two experimental conditions: personalised and control, which were compared in terms of discomfort. The mean ratings for discomfort variables were generally low for both conditions and no significant differences were detected between conditions. In general, the personalisation process showed promise in terms of the scan data, although the foot capture position may not be considered 'gold standard'. Polyamide, the material used for the insoles, demonstrated positive attributes: visual inspection revealed no signs of breaking. The footwear personalisation process described and explored in this study shows potential and can be considered a good starting point for designer and researchers.
\end{abstract}

Keywords: 3D scanning, anthropometry, footwear

\section{Introduction}

The personalisation of footwear is potentially advantageous not only for runners, but to anyone who wishes to become more active, by providing optimum fit, improved comfort and performance. It can also reduce injury risk through personalisation of cushioning and support requirements. Additive manufacturing (AM) technology has potential for making footwear personalisation economically feasible by allowing geometric freedom, its tool-less capabilities and the ability to directly manufacture from CAD models [3]. However, it is not known how to specify such footwear for AM or how personalisation might affect foot discomfort. Therefore, research has been conducted to explore a process that delivers insoles for personalised footwear for the high street using AM and to evaluate the use of such insoles in terms of discomfort.

\section{Footwear personalisation process}

In order to develop a process for the design and manufacture of glove fit personalised insoles, four elements were identified:

- $\quad$ Foot capture using a 3-D scanner;

- Anthropometric measurements;

- Insole design using CAD software;

- Manufacture of the parts using AM technology. This process has the potential to offer footwear with optimum fit, comfort and support properties for a particular individual, but further exploration and evaluation is required.

\footnotetext{
${ }^{*}$ Corresponding author. E-mail: andre_salles@hotmail.com
} 


\section{Evaluation of the process}

\subsection{Sampling}

In order to evaluate the process, six participants $(3$ males and 3 females) were recruited (age $30.5 \mathrm{yrs}, \pm$ 3.9 ; mass $65.25 \mathrm{~kg}, \pm 16.5$; height $165.8 \mathrm{~cm}, \pm 12.8$ ). Sampling criteria were: 18 to 65 years old; to run at least 5 kilometres per week; have no reported musculoskeletal pain or injury in the last 12 months; and to have not used an orthosis in the last 12 months. A repeated measures experimental design was utilized. The study was approved by Loughborough University's Ethical Committee.

\subsection{Procedure}

Trainers from a well-known manufacturer were used and the process was evaluated by comparing two conditions, control (standard shoe + standard insole) and personalised (standard shoe + personalised insole) through single blind trials, whereby a microporous polyurethane foam was used to cover both insoles (Figure 1). A repeated measures experimental design with participants was utilised.

The control condition consisted of the original insole fitted to the standard shoe. The personalised insole was developed following the personalisation process described previously.

The plantar surfaces of the participants' feet were captured in a non-weight bearing position, using a 3dimensional laser scanner (model: eScan 200; 3D Digital Corp, Newtown, CT, USA).

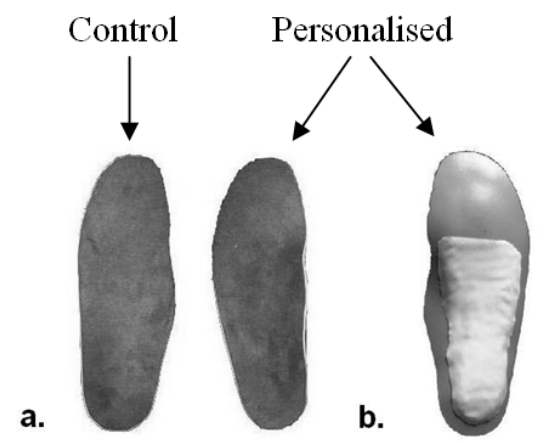

Fig 1. The two experimental conditions used in the study. a: top view; b. bottom view.

Scans were taken with the participants seated with their knee fully extended and their foot dorsiflexed, so that the plantar surface of the foot was parallel to the glass of the scanner. This position was chosen to suit the type of scanner used.

Following the geometry capture of the plantar surface of the foot using the scanner, 15 anthropometric measurements were taken manually following Hawes and Sovak [2] and Williams and McClay [13] to capture dimensional aspects of the foot. These consisted of heights, lengths, widths and girths. Calculations enabled the classification of individuals according to the medial longitudinal arch as follows: arch ratio, arch index and relative arch deformation (RAD) $[6,13]$.

The control insoles were made of a flexible foam material and matched the inside of the footwear from the heel counter to the toe box region. The insoles for the personalised condition were designed to match the form of the participants' feet (glove fit) from the heel to the base of the metatarsal heads and therefore provide support from the heel to the base of the first metatarsal head, but no correction of any lower limb or gait abnormalities. Hence, data manipulation was performed on the scan data solely to rectify and delete unwanted data, but preserving the geometry of the scans. Thus, foot scan data were 'cleaned' to remove any 'noise' and unwanted data, smoothed, thickened to a depth of $3 \mathrm{~mm}$ and converted in to an STL file using Magics software (version: 12.0.0.19; Materialise Group, Leuven, Belgium). Once the parts were designed, they were manufactured using laser sintering (LS) and DuraForm ${ }^{\circledR}$ PA. This material is a rigid nylon (Nylon 12) and its hardness is classified as Shore D 73 [1]. This was chosen for its durability and because it is the most common in LS, so it is easiest to process and more widely known in the research community.

The personalisation process was evaluated according to: compatibility of the data taken from the foot scans (i.e. if the files worked in the reverse engineering software), the capability of the software to manipulate data files, and compatibility of the final data with the AM machines. In addition, the durability of the material (DuraForm ${ }^{\circledR}$ PA) used to manufacture the insoles was examined visually. The position adopted to scan the foot and the usefulness of the anthropometric measurements taken to design and specify the insoles were also evaluated. Finally, the time required to execute each element of the personalisation process was assessed.

To evaluate discomfort in the footwear, participants were asked to run on a treadmill for six minutes at a self-selected speed wearing the trainers fitted with the insoles (randomly assigned) and, after that time, they were given a $150 \mathrm{~mm}$ Visual Analog Scale 
(VAS) to measure self-perceived discomfort. The VAS was similar to one used by Mundermann et al. [4], with the left of the scale indicating 'the most comfortable condition imaginable' and the right 'not comfortable at all'. Six aspects of the foot were evaluated: overall (whole foot), forefoot, midfoot, heel, arch and fit.

\subsection{Analysis}

Significant differences $(\mathrm{p} \leq 0.05)$ between the two conditions were evaluated using a paired samples Student's $t$-test. Pearson's correlation coefficient was used to detect relationships between the anthropometric data and the discomfort. Statistical Package for the Social Sciences (SPSS) software for Windows (Release 15.0, SPSSC, Inc., 2006) was used for all analyses.

\section{Results}

In general, the mean ratings for foot discomfort variables were low for both conditions (Figure 2), but statistical analysis showed no significant differences between the two conditions $(p>0.05)$ for any of the aspects assessed.

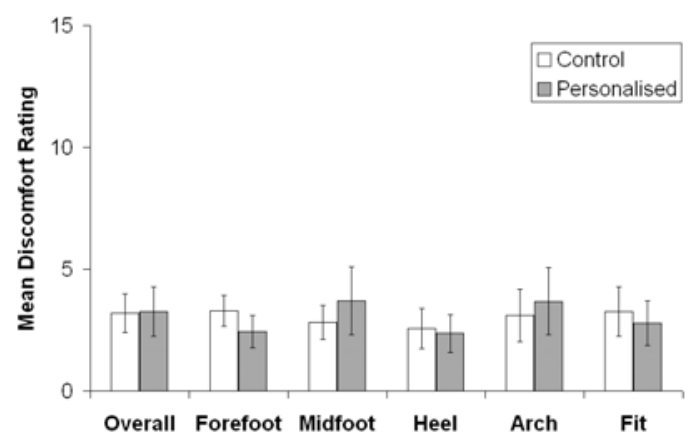

Fig 2. Mean discomfort ratings and standard error for the control and personalised conditions $(n=6)$.

The personalisation process showed promise in terms of the scan data, with scanning taking around 13 seconds to capture the foot.

The foot was captured in a non-weight bearing position to suit the scanner and positioning the foot took on average 2 minutes.

The 15 anthropometric measurements of the foot were taken by the same investigator for each participant in a time span of approximately 25 minutes. Only the anthropometric measurements from the heel to the 1 st and 5 th metarsophalangeal joints (MPJ) were important in the design of the insoles by identifying their end points. In addition to these, only three (MPJ height, hallux height, RAD) proved to be directly useful for specifying the design of the insoles, from the 15 measurements taken.

The whole phase of insole design took a total of two hours per pair. The point-cloud data generated from the scans were compatible with the Magics software. Nevertheless, even though this software enabled the additive manufacturing of parts (by allowing the creation of triangles, repairs, etc.), it did not provide all of the features required for the design of the insoles, especially for smoothing the surface and evening out the jagged edges on the boundaries of the data.

The fabrication time for the 6 pairs of insoles used in the present trials was 28 hours, of which 12 hours were required to actually build the parts and another 12 hours for machine cool down. DuraForm PA, the material used to produce the insoles, demonstrated positive attributes: no sign of breaking was indicated and no significant discomfort reported (compared to the control condition).

\section{Discussion}

Discomfort ratings were low and no significant differences were found between the two conditions. This suggests that the way the personalised insoles were designed and manufactured did not reduce or cause significant discomfort in comparison with the standard ones for a short period of running. The literature is more positive about insoles. For example, Yung-Hui and Wei-Hsien [14] showed that total contact inserts are effective in reducing discomfort when wearing high-heeled shoes. In runners, Mundermann et al. [5] reported that custom made orthotics presented more comfort in comparison to a control condition.

In the present study, the scanner utilised had only one camera, so it provided just a plantar image of the foot, which would not be suitable for the design of the entire shoe. Although the foot posture adopted in the present study may not be considered 'gold standard', the insoles did not cause significant discomfort in comparison to the standard ones. Also, there is no consensus in the literature with regard to the optimal position. Ideally, foot scanners used to design personalised footwear should represent the dynamic movement of the foot, as the foot changes significantly in shape during the ground contact [11]. There are recent scanners that capture a maximum of 49 
frames per second [9], allowing the generation of point cloud data for the different phases of ground contact. However, these systems are still expensive and exclusive and their application to footwear design remains unknown. In this light, further studies are needed to explore the ideal foot position for static foot scans.

The Magics software used in this study is primarily for STL repair and build set-up, but it was the only software available to this research. It is possible that the Geomagic Studio (Geomagic Inc., USA) or Magics CAD (Materialise Group, Belgium) programs used in other studies for reverse engineering can provide a more complex range of resources and thus be more appropriated for this type of data manipulation $[7,10,12]$. However, no literature was found by the authors comparing different computer software for reverse engineering. The phase of insole design using CAD software took a total of two hours per pair. Although this time appears excessive, the researcher has limited experience, such that it may be possible to reduce this time. Tuck et al. [12] reported that the data manipulation phase of customised aircrew seat manufacture takes approximately $20 \%$ of the entire process, whereas in the present study, the same operation took approximately $30 \%$. On top of the LS costs (which include a technician), the data manipulation phase requires a $\mathrm{CAD}$ specialist to design the insoles or footwear for manufacture, making this possibly the most expensive part of the process. In a similar process to the one described here, Pallari et al. [7] included a clinical evaluation by a podiatrist in order to use AM to mass customise orthoses for rheumatoid arthritis suffers. Approximately $£ 50$ a pair has been estimated by Saleh and Dalgarno [8] to produce foot orthoses using Selective Laser Sintering.

\section{Conclusion}

In conclusion, the footwear personalisation process described and explored in this study shows potential and can be considered a good starting point for designer and researchers. The scan data files were compatible with all hardware and software utilised throughout the process and the insoles did not cause significant discomfort in comparison to the commercially available ones, indicating that manufacturing personalised insoles via foot scans and AM is feasible. This study was a pre-cursor for a longitudinal trial involving a broader sample of the population.

\section{References}

[1] 3D Systems, DuraForm ${ }^{\circledR}$ PA plastic for use with all selective laser sintering (SLS $®)$ systems [online]. Available from: www.3dsystems.com/products/datafiles/lasersintering/datashe ets/DS-DuraForm_PA_plastic-A4_UK.pdf [accessed May 2010].

[2] M.R. Hawes and D. Sovak, Quantitative morphology of the human foot in a North American population, Ergonomics 37 (1994), 1213-1226.

[3] N. Hopkinson and P. Dickens, Analysis of rapid manufacturing - using layer manufacturing processes for production, Proceeding of the Institution of Mechanical Engineers, Part C: Journal of Mechanical Engineering Science 217 (2003), 31-39.

[4] A. Mundermann, B.M. Nigg, D.J Stefanyshyn and R.N Humble, Development of a reliable method to assess footwear comfort during running, Gait and Posture 16 (2002), 38-45.

[5] A. Mundermann, B.M. Nigg, R.N. Humble, D.J. Stefanyshyn, Orthotic comfort is related to kinematics, kinetics and EMG in recreational runners, Medicine and Science in Sports and Exercise 35 (2003), 1710-1719.

[6] B.M. Nigg, A. Khan, V. Fisher and D.J. Stefanyshyn, Effect of shoe insert construction on foot and leg movement, Medicine and Science in Sports and Exercise 30 (1998), 550-555.

[7] J.H.P. Pallari, K.W. Dalgarno and J. Woodburn, Mass customisation of foot orthoses for rheumatoid arthritis using selective laser sintering, IEEE Transactions on Bio-medical Engineering 57 (2010), 1750-1756.

[8] J.M. Saleh and K.W. Dalgarno, Cost and benefit of Fused Deposition Modelling (FDM) technique and Selective Laser Sintering (SLS) for fabrication of customised foot orthoses, in: Innovative Developments in Design and Manufacturing: Advanced research in virtual and rapid prototyping, P.J.S. Bártolo, et al., eds., Taylor and Francis, London, 2010, pp. 705-710.

[9] T. Schmeltzpfenning, C. Plank, I. Krauss, P. Aswendt and S. Grau, Dynamic foot scanning. Prospects and limitations using synchronized 3D scanners to capture complete human foot shape while walking, in: Proceedings of the 3rd International Conference on Applied Human Factors and Ergonomics, Miami, USA, 2010.

[10] S.P. Sun, Y.J. Chou and C.C. Sue, Classification and mass production technique for three-quarter shoe insoles using nonweight-bearing plantar shapes, Applied Ergonomics 40 (2009), 630-635.

[11]B.Y.S. Tsung, M. Zhang, Y.B. Fan and D.A. Boone, Quantitative comparison of plantar foot shapes under different weightbearing conditions, Journal of rehabilitation R\&D 40 (2003), 517-526.

[12] C.J. Tuck, R.J.M. Hague, M. Ruffo, M. Ransley and P. Adams, Rapid manufacturing facilitated customization, International Journal of Computer Integrated Manufacturing 21 (2008), 245-258.

[13]D.S. Williams and I.S. McClay, Measurements used to characterize the foot and the medial longitudinal arch: reliability and validity, Physical Therapy 80 (2000), 864-871.

[14]L. Yung-Hui and H. Wei-Hsien, Effects of shoe inserts and heel height on foot pressure, impact force and perceived comfort during walking, Applied Ergonomics 36 (2005), 355-362. 